\title{
INTEGRAL MONSKY-WASHNITZER COHOMOLOGY AND THE OVERCONVERGENT DE RHAM-WITT COMPLEX
}

\author{
Christopher Davis and David Zureick-Brown
}

\begin{abstract}
In their paper, which introduced Monsky-Washnitzer cohomology, Monsky and Washnitzer described conditions under which the definition can be adapted to give integral cohomology groups. It seems to be well-known among experts that their construction always gives well-defined integral cohomology groups, but this fact also does not appear to be explicitly written down anywhere. In this paper, we prove that the integral Monsky-Washnitzer cohomology groups are well-defined, for any nonsingular affine variety over a perfect field of characteristic $p$. We then compare these cohomology groups with overconvergent de Rham-Witt cohomology. It was shown earlier that if the affine variety has small dimension relative to the characteristic of the ground field, then these cohomology groups are isomorphic. We extend this result to show that for any nonsingular affine variety, regardless of dimension, we have an isomorphism between integral Monsky-Washnitzer cohomology and overconvergent de Rham-Witt cohomology in degrees which are small relative to the characteristic.
\end{abstract}

\section{Introduction}

Monsky-Washnitzer cohomology is usually defined rationally, but cases in which it could be defined integrally were already given in Monsky and Washnitzer's original paper [7, Remark 3, p. 205]. The integral definition for arbitrary nonsingular affine varieties (and in particular the fact that it is well-defined) does not seem to be written down formally anywhere, and so we describe it below.

Overconvergent de Rham-Witt cohomology was studied in [1]. It was shown there that, rationally, it agreed with Monsky-Washnitzer cohomology. Conditions were given on the nonsingular affine variety $\bar{X}$ under which the Monsky-Washnitzer cohomology groups were isomorphic to the integral overconvergent de Rham-Witt cohomology groups [1, Corollary 3.25(a)]. In this paper, we extend the result to show that certain of these cohomology groups are always isomorphic, without any conditions on the nonsingular affine variety $\bar{X}$. In particular, there is an integral comparison isomorphism in degrees 0 and 1.

Our main results are the following. The authors suspect that the first is already known to experts. (See the end of this section for our notation and conventions.)

Theorem 1.1. Let $\bar{X}=\operatorname{Spec} \bar{A}$ denote a nonsingular affine variety over a perfect field $k$ of characteristic $p$. Let $A^{\dagger}$ denote the weak completion of a nonsingular lift to $W(k)$.

(1) The integral Monsky-Washnitzer cohomology groups $H^{i}\left(\Omega_{A^{\dagger} / W(k)}\right)$ are well-defined. More precisely, given two lifts $A$ and $A^{\prime}$ of $\bar{A}$, there exists an

Received by the editors April 30, 2013. 
isomorphism

$$
H^{i}\left(\Omega_{A^{\dagger} / W(k)}^{\bullet} \stackrel{\sim}{\longrightarrow} H^{i}\left(\Omega_{A^{\prime \dagger} / W(k)}^{\bullet}\right) .\right.
$$

(2) We have an isomorphism

$$
H^{i}\left(\Omega_{A^{\dagger} / W(k)}^{\bullet} \stackrel{\sim}{\longrightarrow} H^{i}\left(W^{\dagger} \Omega \cdot \frac{\bullet}{A}\right)\right.
$$

for all $i<p$.

Proof. The proof of (1) is given in [7, Remark 3, p. 205] in the special case that $\bar{X}$ is a complete transversal intersection. For the proof in the general case, see Section 2 below. The key pieces to the proof are the local result of Monsky and Washnitzer just referenced, a result of Meredith concerning sheaf properties of the Monsky-Washnitzer complex, and a Čech spectral sequence argument.

The proof of (2) is given in Section 3. Again the strategy of the proof is to prove the result locally, which we do using results from [1], and then to deduce the result in general using a Čech spectral sequence argument.

Notation. Let $k$ denote a perfect field of characteristic $p$ and let $W(k)$ denote the ring of $p$-typical Witt vectors with coefficients in $k$. By variety over $k$, we mean a separated and integral scheme of finite type over the (perfect but not necessarily algebraically closed) field $k$. Throughout this paper, let $\bar{A}$ denote the coordinate ring of a nonsingular affine variety over $k$. We write $\bar{X}=\operatorname{Spec} \bar{A}$.

\section{Integral Monsky-Washnitzer cohomology}

Let $\bar{X}=$ Spec $\bar{A}$ denote a nonsingular affine variety over the perfect field $k$ of characteristic $p$. We begin by reproducing Monsky and Washnitzer's construction of the Monsky-Washnitzer cohomology groups associated to $\bar{A}$, with the key difference that we never tensor with $\mathbb{Q}$. By $[2$, Theorem 6] we can lift $\operatorname{Spec} \bar{A}$ (in many ways) to an affine variety which is smooth over Spec $W(k)$. For such a lift $\operatorname{Spec} A$, define the weak completion $A^{\dagger}$ and the complex of continuous differentials $\Omega_{A^{\dagger} / W(k)}^{\bullet}$ as in $[7$, Definition 1.1 and Theorem 4.2].

Definition 2.1. The (integral) Monsky-Washnitzer cohomology groups are $H_{\mathrm{MW}}^{i}(\operatorname{Spec} \bar{A}):=H^{i}\left(\Omega_{A^{\dagger} / W(k)}^{\bullet}\right)$.

The goal of this section is to show that Definition 2.1 does not depend on a choice of lift $A$. We first note that, although the correspondence $\bar{A} \rightsquigarrow A^{\dagger}$ is not functorial, we can at least lift maps.

Proposition 2.2. Let $\bar{\varphi}: \bar{A} \rightarrow \bar{B}$ denote a ring homomorphism. Choose $A, B$, two lifts to characteristic 0 as above, and let $A^{\dagger}, B^{\dagger}$ denote the corresponding weak completions. Then there exists a (typically not unique) p-adically continuous ring homomorphism $\varphi: A^{\dagger} \rightarrow B^{\dagger}$ lifting $\varphi$.

Proof. This follows from [6, Proposition 1.3] and [8, Theorem 2.4.4].

The following relies on the notion of a complete transversal intersection as defined in [7, Definition 3.3]. 
Proposition 2.3. Let $\operatorname{Spec} \bar{A}$ denote a complete transversal intersection. Let $\operatorname{Spec} A$, Spec $A^{\prime}$ denote two nonsingular lifts of $\operatorname{Spec} \bar{A}$, and let $A^{\dagger}, A^{\prime \dagger}$ denote the corresponding dagger algebras. Let $\varphi: A^{\dagger} \rightarrow A^{\prime \dagger}$ denote a map lifting the identity on $\bar{A}$, as in Proposition 2.2. Then the induced map

$$
\varphi: \Omega_{A^{\dagger} / W(k)}^{\bullet} \rightarrow \Omega_{A^{\prime \dagger} / W(k)}^{\bullet}
$$

is a quasi-isomorphism.

Proof. See [7, Remark 3, p. 205].

We will prove well-definedness of integral Monsky-Washnitzer cohomology in general using the special case of complete transversal intersections. First we interpret the result of Proposition 2.3 as a local condition, and then we "glue" using certain sheaf properties which are provided by the work of Meredith.

Lemma 2.4. Let $\bar{X}$ denote a nonsingular affine variety over $k$. Then $\bar{X}$ can be covered by finitely many complete transversal intersections in such a way that all finite intersections are also complete transversal intersections.

Proof. We can cover $\bar{X}$ by complete transversal intersections by the Jacobian criterion. The result now follows by taking a suitable refinement by distinguished opens, as in for example [3, Proposition 6.3.2].

Definition 2.5. Let $\mathscr{F}_{A^{\dagger}}^{i}$ denote the presheaf on $\operatorname{Spec} \bar{A}$ associated to $\Omega_{A^{\dagger} / W(k)}^{i}$ as in [6, Definition 4].

Lemma 2.6. The presheaf $\mathscr{F}_{A^{\dagger}}^{i}$ is a sheaf. For any $i$ and for any $j>0$, we have $H^{j}\left(\operatorname{Spec} \bar{A}, \mathscr{F}_{A^{\dagger}}^{i}\right)=0$.

Proof. This follows from [7, Theorem 4.5] and [6, Theorem 14].

We now come to the proof that integral Monsky-Washnitzer cohomology is welldefined.

Proof of Theorem 1.1(1). By Proposition 2.2, there exists a map $\varphi: A^{\dagger} \rightarrow A^{\prime \dagger}$ lifting the identity on $\bar{A}$. Fix a covering $\mathscr{U}$ of $\operatorname{Spec} \bar{A}$ as in Lemma 2.4. Let $E$ (resp., $E^{\prime}$ ) denote the Čech cohomology spectral sequence corresponding to the covering $\mathscr{U}$ and the complex of sheaves $\mathscr{F}_{A^{\dagger}}^{\bullet}$ (resp., $\mathscr{F}_{A^{\prime \dagger}}^{\bullet}$ ). By Proposition 2.3, the map $\varphi$ induces a map of spectral sequences and isomorphisms $E_{2}^{p, q} \stackrel{\sim}{\longrightarrow} E_{2}^{\prime p, q}$ for all $p, q$. Thus the map $\varphi$ induces an isomorphism $E_{\infty}^{p+q} \stackrel{\sim}{\longrightarrow} E_{\infty}^{\prime p+q}$ and furthermore the induced map

$$
\varphi: \mathbb{H}^{p+q}\left(X, \mathscr{F}_{A^{\dagger}}^{\bullet}\right) \rightarrow \mathbb{H}^{p+q}\left(X, \mathscr{F}_{A^{\prime \dagger}}^{\bullet}\right)
$$

is an isomorphism. By Lemma 2.6, these hypercohomology groups agree with the integral Monsky-Washnitzer cohomology groups, which completes the proof. 


\section{Comparison with overconvergent de Rham-Witt cohomology}

In this section, we prove Theorem 1.1(2). The benefit of describing MonskyWashnitzer cohomology groups in terms of the overconvergent de Rham-Witt complex is that this latter complex is completely functorial, and does not depend on a choice of lift to characteristic zero.

As in Section 2 , let $\bar{X}=\operatorname{Spec} \bar{A}$ denote a nonsingular affine variety over a perfect field $k$ of characteristic $p$. We will first define a (non-canonical) comparison morphism between the integral Monsky-Washnitzer complex $\Omega_{A^{\dagger} / W(k)}$ and the overconvergent de Rham-Witt complex $W^{\dagger} \Omega_{\bar{A}}^{\bullet}$. We will then find a covering of $\bar{X}$ over which this comparison morphism induces an isomorphism of certain cohomology groups. Lastly we will use the Cech cohomology spectral sequence to deduce the result for $\bar{X}$.

Let Spec $\bar{A}$ denote a nonsingular affine variety, and let $A^{\dagger}$ denote a weakly complete lift of $\bar{A}$, as in Section 2. By Proposition 2.2, there exists a lift of Frobenius $F: A^{\dagger} \rightarrow A^{\dagger}$.

Proposition 3.1. Let $\bar{A}, A^{\dagger}, F$ be as above. There exists a unique ring homomorphism

$$
s_{F}: A^{\dagger} \rightarrow W\left(A^{\dagger}\right)
$$

such that, for any $a \in A^{\dagger}$, the ghost components of $s_{F}(a)$ are $\left(a, F(a), F^{2}(a), \ldots\right)$. This map is functorial in the sense that if we have $\bar{A}^{\prime}, A^{\prime \dagger}, F^{\prime}$ as above and a map $\varphi$ such that the left-hand square in the following diagram commutes, then the right-hand square also commutes:

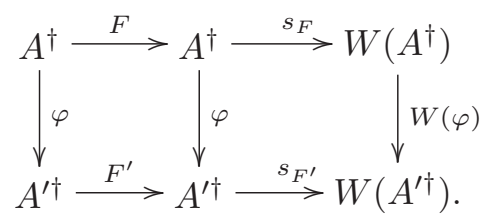

Proof. See [4, (0.1.3.16)].

Definition 3.2. Keep notation as above. Let $W^{\dagger} \Omega_{\bar{A}}^{\bullet}$ denote the overconvergent de Rham-Witt complex, as in [1, Definition 1.1] and let $W^{\dagger} \Omega_{\bar{X}}^{\bullet}$ denote the associated complex of Zariski sheaves on $X$ as in [1, Corollary 1.6]. Write $t_{F}$ for the composition $A^{\dagger} \stackrel{s_{F}}{\longrightarrow} W\left(A^{\dagger}\right) \rightarrow W^{\dagger}(\bar{A})$, as in [1, Proposition 3.2]. Also write $t_{F}$ for the induced map $\Omega_{A^{\dagger} / W(k)}^{\bullet} \rightarrow W^{\dagger} \Omega_{\dot{A}}^{\bullet}$.

Proposition 3.3. Let $\bar{A}, A^{\dagger}, F$ be as above and let $\mathscr{F}_{A^{\dagger}}^{\bullet}$ be as in Definition 2.5. Then the map $t_{F}$ defined in Definition 3.2 induces a map on complexes of Zariski sheaves

$$
t_{F}: \mathscr{F}_{A^{\dagger}}^{\bullet} \rightarrow W^{\dagger} \Omega \bullet_{\bar{A}}^{\bullet}
$$

Proof. Because the maps on complexes are determined by the maps in degree zero, it suffices to prove this in degree zero. For any $f \in A^{\dagger}$, let $A_{[f]}^{\dagger}$ denote the weak completion of the localization $A_{f}^{\dagger}$, as in [6, Definition 2.1]. The $p$-adically continuous ring homomorphism $F: A^{\dagger} \rightarrow A^{\dagger}$ extends uniquely to a $p$-adically continuous ring homomorphism $A_{[f]}^{\dagger} \rightarrow A_{[f]}^{\dagger}$. Hence we are finished by the functoriality assertion in Proposition 3.1. 
Our goal is to show that the comparison map $t_{F}$ defined above induces an isomorphism between (certain) integral Monsky-Washnitzer cohomology groups and (certain) overconvergent de Rham-Witt cohomology groups. This result will generalize [1, Corollary 3.25(a)], which states that if the dimension of $\bar{X}$ is small relative to the characteristic $p$, then these comparison maps induce isomorphisms between (all) integral Monsky-Washnitzer cohomology groups and (all) overconvergent de RhamWitt cohomology groups.

Our strategy for showing that these comparison maps induce isomorphisms in certain degrees will be to prove the result locally, and then deduce the result in general using the Čech spectral sequence. The Čech covering of $\bar{X}$ we use will typically be finer than the covering used in Section 2.

Definition 3.4. An affine variety is called special if it is a distinguished open inside a scheme which is finite étale over affine space.

Proposition 3.5. Let $\bar{X}$ denote a nonsingular affine variety over $k$. Then $\bar{X}$ can be covered by finitely many special affines in such a way that all finite intersections are also special.

Proof. The fact that $\bar{X}$ can be covered by special affines comes from [5]. Note that a distinguished open inside of a special affine is also a special affine. We are now finished as in the proof of Lemma 2.4 .

Lemma 3.6. Let $\bar{X}$ denote a nonsingular affine variety over $k$. Then $\bar{X}$ can be covered by finitely many open affines which are both special and complete transversal intersections, and in such a way that any finite intersection is also both special and a complete transversal intersection.

Proof. Begin by finding two separate coverings, one as in Proposition 3.5 and one as in Lemma 2.4. These are both coverings by distinguished opens; in one case the distinguished opens are special affines and in the other case they are complete transversal intersections. Because distinguished opens of special affines (resp., complete transversal intersections) are special affines (resp., complete transversal intersections), intersecting these two coverings completes the proof.

The following proposition asserts that integral Monsky-Washnitzer cohomology is isomorphic to overconvergent de Rham-Witt cohomology in the particular case that $\bar{X}=\operatorname{Spec} \bar{A}$ is a special affine. It makes use of a certain comparison map $\sigma$ defined in [1]; the definition depends on a choice of presentation of $\bar{A}$.

Proposition 3.7. Let $\bar{X}=\operatorname{Spec} \bar{A}$ denote a special affine. Fix both a weakly complete lift $A^{\dagger}$ and a comparison map

$$
\sigma: \Omega_{A^{\dagger} / W(k)}^{\bullet} \rightarrow W^{\dagger} \Omega_{\frac{\bullet}{A}}
$$

as in [1, (3.5)]. For all $i$, the induced map

$$
\sigma: H^{i}\left(\Omega_{A^{\dagger}}^{\bullet} \rightarrow H^{i}\left(W^{\dagger} \Omega_{\frac{\bullet}{A}}\right)\right.
$$

is an isomorphism.

Proof. See [1, Theorem 3.19]. 
Note that Proposition 3.7 asserts an isomorphism between the cohomology groups in all degrees, but it requires a particular choice of comparison map $\sigma$. For $\bar{X}$ arbitrary, not necessarily special affine, there is not an obvious analogue of $\sigma$. For this reason, below we will take for our comparison map $t_{F}$, as in Definition 3.2. Upon restricting to a special affine, we are only able to prove that the maps induced on cohomology by $\sigma$ and $t_{F}$ agree in suitably small degrees. This explains the restriction on degree in Theorem 1.1(2).

Proposition 3.8. Let $\bar{X}=\operatorname{Spec} \bar{A}$ denote a special affine. Fix a weakly complete lift $A^{\dagger}$ as in Section 2, and fix a lift of Frobenius $F: A^{\dagger} \rightarrow A^{\dagger}$. Let

$$
t_{F}: \Omega_{A^{\dagger} / W(k)}^{\bullet} \rightarrow W^{\dagger} \Omega_{\bar{A}}^{\bullet}
$$

be the comparison morphism from Definition 3.2. Then for all $i<p$, the induced map

$$
t_{F}: H^{i}\left(\Omega_{A^{\dagger} / W(k)}^{\bullet}\right) \rightarrow H^{i}\left(W^{\dagger} \Omega_{\frac{\bullet}{A}}\right)
$$

is the same as the isomorphism $\sigma$ from Proposition 3.7. In particular, the maps on cohomology induced by $t_{F}$ are independent of the choice of Frobenius lift $F$.

Proof. We deduce this from the proof of [1, Proposition 3.27] as follows. Let

$$
\phi_{1}, \phi_{2}: \Omega_{A^{\dagger} / W(k)}^{\bullet} \rightarrow W^{\dagger} \Omega_{\bar{A}}^{\bullet}
$$

denote two morphisms of differential graded algebras which induce the identity map on $\bar{A}$; we will apply this to the two maps $\sigma$ and $t_{F}$. Choose $i<p$ and let $\omega \in \Omega_{A^{\dagger} / W(k)}^{i}$ denote a cocycle. We would like to show that $\phi_{1}(\omega)-\phi_{2}(\omega)$ is a coboundary in $W^{\dagger} \Omega_{\frac{i}{A}}$.

We claim that for $i<p$ and for the maps $\varphi, L$ defined in the proof of [1, Proposition 3.27], the map

$$
L \circ \varphi: \Omega_{A^{\dagger} / W(k)}^{i} \rightarrow W^{\dagger} \Omega_{\bar{A}}^{i-1}
$$

is such that

$$
d(L \circ \varphi(\omega))=\phi_{1}(\omega)-\phi_{2}(\omega)
$$

By the proof of [1, Proposition 3.27], we need only to check that the map $L \circ \varphi$ does indeed have image in $W^{\dagger} \Omega_{\frac{i-1}{A}}$. (The proof in [1] shows this only after multiplying by $p^{\kappa}$, for $\kappa=\left\lfloor\log _{p} \operatorname{dim} \bar{X}\right\rfloor$.) By [1, Lemma 3.31(i)] and the definition of $L$, we are reduced to proving that if $\omega_{j} \in W^{\dagger} \Omega_{\frac{i-1}{A}}^{i}$ is divisible by $p^{\max (j-i+1,0)}$ for some $j \geq 0$, then

$$
\frac{\omega_{j}}{j+1} \in W^{\dagger} \Omega_{\bar{A}}^{i-1} \text {. }
$$

If $\max (j-i+1,0)=0$, then $j+1 \leq i<p$, and the result is trivial, since $j+1$ is a unit in $W(k)$. So it remains to consider the case $\max (j-i+1,0)=j-i+1>0$. The first case in which this occurs is $j=i$. Recalling that $i<p$, we are finished in this case because $i-i+1=1 \geq \log _{p}(i+1)$. The remaining cases are also easy, because $j-i+1$ grows faster with $j$ than $\log _{p}(j+1)$.

To prove Theorem 1.1(2), we will again use the Čech spectral sequence. To account for the restriction on degrees in Proposition 3.8, we need the following result. 
Lemma 3.9. Fix $n \geq 0$. Let $f: E \rightarrow \widetilde{E}$ be a morphism of first quadrant spectral sequences such that the induced map $f: E_{2}^{p, q} \rightarrow \widetilde{E}_{2}^{p, q}$ is an isomorphism for all $p$ and all $q \leq n$. Then the induced map $E_{\infty}^{p+q} \rightarrow \widetilde{E}_{\infty}^{p+q}$ is an isomorphism for $p+q \leq n$.

Proof. For each $i$ and $p+q \leq n$, the hypotheses and an inspection of the domain and codomain of the differentials imply that the map $E_{i}^{p, q} \rightarrow \widetilde{E}_{i}^{p, q}$ is an isomorphism.

We are now ready to prove the second half of our main theorem.

Proof of Theorem 1.1(2). Fix a weakly complete lift $A^{\dagger}$ of $A$, and a lift of Frobenius $F: A^{\dagger} \rightarrow A^{\dagger}$. Let $\mathscr{F}_{A^{\dagger}}^{\bullet}$ denote the complex of sheaves associated to $A^{\dagger}$ as in Definition 2.5. Let

$$
t_{F}: \mathscr{F}_{A^{\dagger} / W(k)}^{\bullet} \rightarrow W^{\dagger} \Omega{ }_{\bar{A}}
$$

denote the morphism of sheaves, as in Proposition 3.3. Now choose a distinguished open covering $\mathscr{U}$ of $\operatorname{Spec} \bar{A}$ as in Lemma 3.6. Let $E$ (resp., $E^{\prime}$ ) denote the Cech cohomology spectral sequence corresponding to the covering $\mathscr{U}$ and the complex of sheaves $\mathscr{F}_{A^{\dagger}}^{\bullet}$ (resp., $W^{\dagger} \Omega_{\bar{X}}^{\bullet}$ ). Let $U_{I}$ denote an arbitrary finite intersection of opens $U_{\alpha} \in \mathscr{U}$ and consider the induced map on cohomology

$$
t_{F}: \mathbb{H}^{i}\left(U_{I}, \mathscr{F}_{A^{\dagger}}^{\bullet}\right) \rightarrow \mathbb{H}^{i}\left(U_{I}, W^{\dagger} \Omega_{\frac{\bullet}{A}}\right) .
$$

By Lemma 2.6 (resp., [1, Proposition 1.2(b)]) the individual sheaves in the complex $\mathscr{F}_{A^{\dagger}}^{\bullet}$ (resp., $W^{\dagger} \Omega_{\bar{X}}^{\bullet}$ ) have trivial sheaf cohomology. Hence Proposition 3.8 implies that the maps $t_{F}$ above are isomorphisms for $i<p=$ Char $\bar{A}$. (Our reason to avoid the letter $p$ will be clear in the next paragraph.)

Thus $t_{F}$ induces a map of spectral sequences and isomorphisms $E_{2}^{p, q} \stackrel{\sim}{\longrightarrow} E_{2}^{\prime p, q}$ for all $p$ and all $q<$ Char $\bar{A}$. Then by Lemma 3.9 the map $t_{F}$ induces an isomorphism $E_{\infty}^{p+q} \stackrel{\sim}{\longrightarrow} E_{\infty}^{\prime p+q}$ for $p+q<$ Char $\bar{A}$. Hence the induced map

$$
t_{F}: \mathbb{H}^{p+q}\left(X, \mathscr{F}_{A^{\dagger}}^{\bullet}\right) \rightarrow \mathbb{H}^{p+q}\left(X, W^{\dagger} \Omega_{\bar{X}}^{\bullet}\right)
$$

is an isomorphism for $p+q<\operatorname{Char} \bar{A}$. These hypercohomology groups agree with the integral Monsky-Washnitzer and overconvergent de Rham-Witt cohomology groups, which completes the proof.

Remark 3.10. It would be interesting to have an example in which there is not an integral isomorphism between Monsky-Washnitzer cohomology and overconvergent de Rham-Witt cohomology (or to prove that no such example exists). By Theorem 1.1, the dimension of Spec $\bar{A}$ in such an example would have to be at least the characteristic $p$. Perhaps the first class to consider when seeking such an example would be the two-dimensional affine varieties over $\mathbb{F}_{2}$.

\section{Acknowledgments}

This paper answers a question asked by Liang Xiao at the first author's thesis defense. We thank Liang for his interest and for recognizing the opportunity to extend the previous results, and also for a careful reading of an earlier draft. The first author also thanks his earlier coauthors Andreas Langer and Thomas Zink for their support and encouragement. Thanks also to Veronika Ertl and Kiran Kedlaya for many useful conversations and suggestions. The first author is partially supported by the Danish 
National Research Foundation through the Centre for Symmetry and Deformation (DNRF92).

\section{References}

[1] C. Davis, A. Langer and T. Zink, Overconvergent de Rham-Witt cohomology, Ann. Sci. Éc. Norm. Supér. (4) 44(2) (2011), 197-262.

[2] R. Elkik, Solutions d'équations à coefficients dans un anneau hensélien, Ann. Sci. École Norm. Sup. (4) 6 (1973), 553-603 (1974)

[3] A. Grothendieck, Éléments de géométrie algébrique. I. Le langage des schémas, Inst. Hautes Études Sci. Publ. Math. (4) (1960), 5-228

[4] L. Illusie, Complexe de de Rham-Witt et cohomologie cristalline, Ann. Sci. École Norm. Sup. (4) $\mathbf{1 2}(4)$ (1979), 501-661

[5] K.S. Kedlaya, More étale covers of affine spaces in positive characteristic, J. Algebraic Geom. 14(1) (2005), 187-192

[6] D. Meredith, Weak formal schemes, Nagoya Math. J. 45 (1972), 1-38

[7] P. Monsky and G. Washnitzer, Formal cohomology. I, Ann. Math. (2) 88 (1968), 181-217

[8] M. van der Put, The cohomology of Monsky and Washnitzer, Mém. Soc. Math. France (N.S.) (23) (1986), 4, 33-59. Introductions aux cohomologies $p$-adiques (Luminy, 1984)

Department of Mathematics, University of California, Irvine, Irvine, CA 92697, USA Current address: Department of Mathematical Sciences, University of Copenhagen Universitetsparken 5, 2100 København $\varnothing$, Denmark

E-mail address: davis@math.ku.dk

Department of Mathematics and Computer science, Emory University, Atlanta, GA 30322, USA

E-mail address: dzb@mathcs.emory.edu 